\title{
The Development of an Evolutionary Algorithm to Predict Outcomes in Meteorological Trends
}

\author{
Louellen E. McCoy, Stuart H. Rubin \\ SPAWAR System Center-Pacific \\ Louellen.mccoy@navy.mil \\ stuart.rubin@navy.mil
}

\begin{abstract}
This paper addresses the use evolutionary algorithm to accurately predict natural phenomena. Evolutionary algorithms are techniques based on patterns found in nature and are useful in analyzing trends in data. Evolution is an optimization process that finds solutions to problems by employing a set of mutation and reproduction rules over a series of generations. Nonmonotonic reasoning is also an area examined as a possible way to implement the rule-based system. This paper investigates the use of evolutionary algorithms, programmed in Java, to predict outcomes in weather patterns. The tie-in to the prediction of insurgent activities follows.
\end{abstract}

Keywords: Evolutionary Algorithms, Weather, Dynamic Systems, Non-Monotonic Reasoning

\section{Introduction}

Evolutionary algorithms are techniques based on patterns found in nature and are useful in analyzing trends in data. Evolution is an optimization process that finds solutions to problems by employing a set of mutation and reproduction rules over a series of generations [1].Patterned after studies of adaptation processes in biology, it has extended its reach into areas such as computer science and many branches of engineering. Evolutionary algorithms have proven themselves extremely useful in finding estimations of solutions to complex problems where finding an exact solution may be impossible [2]. Work is being accomplished towards an application that reads that data collected from nature and analyzes patterns in the data using an evolutionary algorithm. The GUI and the core of the application will be programmed in Java. The Java programming language is appropriate for this application because of its free distribution, graphical user interface capabilities, prepackaged data structure implementations, and Application
Programming Interfaces (APIs) that can be imported into the environment as plug-in functionality. The use of APIs can save much implementation time.

\section{Algorithm Approach}

The application to be developed is an evolutionary rule-based system for predicting trends in natural phenomena. The goal of the research and development, involved this project, is to mine and develop new rules in an adaptive system. An important and useful application of the development of evolutionary algorithms is in predicting natural phenomena, such as weather forecasting. The system will analyze data from previous time periods and evaluate it to make a prediction for a future time period. These data could be taken from multiple sources, such as archived data from the National Oceanographic and Atmospheric Association (NOAA).

Many commercial, government, media, and academic sources track weather measurements that are key factors in meteorological prediction, such as wind direction, wind speed, barometric pressure, humidity, temperature and wind chill. Many of these weather reports are available from internet sources, news broadcasts, universities, and the government which consolidates the information in reports. The weather features can be stored in an Excel spreadsheet as features in columns. Additional weather features can be calculated from the raw data - such as dynamic changes in some metric. The fact that a certain parameter, such as barometric pressure or temperature, is rising or falling creates data that can be used in weather prediction.

A rule is defined as a combination of a discrete number of features in the antecedent and a result in the consequent. The antecedents of the rules contain the features. The features are the conditions of the weather. A feature could be, for example, "low barometric pressure" or "high temperature." A consequent could be another 
feature or a predefined description such as raining, hailing, snowing, or sunny. The consequent of a rule defines the outcome of a combination of features after a discrete time period.

A set of rules will be established as the base set; then, the rules will evolve. There will be two sets of rules: corrector rules and predictor rules. The term, "corrector rule", will be used to describe rules that change according to appropriate circumstances. For example, there might be a rule that reads, if it is 0 degrees Fahrenheit and low barometric pressure, then it will rain. The actual result should snow because of the low temperature. Therefore, the rule is changed to "it will snow." A predictive rule will look at the data of the scenario and predict the outcome, such as "there is humidity." A sample of data, in this context, is defined as a column in the table of data. The accuracy of the rules can be tested by randomly selecting the column to test.

The individuals in this evolutionary system that represent the basis of the algorithm are the rules that actually evaluate the weather. The rules are evaluated on a blackboard space. Another term for blackboard space is context. The context contains states, which are the conditions of the weather. The rules would then be evaluated on the blackboard to predict the outcome of the weather (e.g., it's raining, it's snowing). The outcome of a rule can be added to the context itself, therefore changing the context. In order to overcome local optima, the algorithm will evolve the rules. The algorithm mutates a defined number of features in the rules subsequent to each iteration; but, the sample for evaluation is taken randomly on each iteration.

There is a set of features that is maintained throughout the duration of the algorithm. The basis of a mutation is the random selection of features that are either changed or deleted. For example, if there are seven features, a random mutation of three features per iteration would be to change the random selection of features 1,3 , and 7 from the previous entry into a different feature or mark the feature for deletion. The rules, where the selected features $(1,3$, or 7$)$ are found would then be swapped out with the new feature selected randomly within a range of enumerated values, or deleted if it is so marked.

Consider a rule with two features. If swapping a feature in the antecedent of a two-featured rule causes a duplication, then the features that cause the consequent would go from two features to one feature in number. For example, If there is a rule that states low barometric pressure and low temperature cause it to rain, and the low barometric temperature feature is swapped out with a feature's newly mutated feature value of "low temperature", then the rule would be changed to "low temperature causes rain." Once the rules are changed, they are evaluated against the weather conditions, with the results being displayed on the blackboard. The effective rules are saved, and the less effective rules are mutated. These procedural steps are the basis for the evolutionary component of the algorithm.

\section{Software Design}

The design of the software which processes the algorithm may be accomplished using several different components performing the processing for important tasks such as data initialization, evaluation of samples, mutation, and measurement of performance. The algorithm analyzes measurements of weather taken over a time sequence. These data can easily be stored either in an Excel spreadsheet or a comma delineated CSV file and retrieved using a JAVA parsing routine or API. Another component of the software is designated to generate the first part of the rules using randomly constructed rules for a set of features.

A significant part of the algorithm is a rule evaluator, which uses a specialized rule evaluation technique called, non-monotonic reasoning. Non-monotonic reasoning provides the capability to have additional knowledge add or subtract what is known from the context. An important aspect of the algorithm is the number of samples and the number of mutations performed during the evolutionary progression. If the number of samples is too large, then it slows down the progression towards the solution. Likewise, it is not efficient to process a large number of mutations for each step of the algorithm. Unnecessary amounts of mutation can actually hinder the convergence towards a global solution.

The sampling process for the algorithm can be summarized with a number of subroutines that are repeated in a cyclical manner. The first generated rule set is evaluated against a randomly selected set of size $n$ and a mutated version is also evaluated against the same randomly selected set of size $n$. The outcome of the trial is measured against the following day's actual weather result from the collection of data. The simplest measure that can be used is the Euclidean distance norm and, using the terminology of evolutionary computation, it is the metric (fitness function), which determines if performance has improved in the current generation.

The component that evaluates the successive generations of rule sets on actual data is divided into subroutines and performed as a series of iterations. A rule set and its mutated version are evaluated on a sample of 
weather data and then both are measured against the actual results on the following days using a Euclidean measure; these steps comprise a subroutine of the algorithm that is repeated in a cycle. The best rule set from the first cycle is stored and then another cycle of the subroutine is computed, where the same rule set and mutated version are evaluated against another randomly selected set of the same size. The best rule set of this cycle is also stored. If, for two cycles in a row, the same rule base has the best performance measured on the actual results using the Euclidean norm, then the sample size is decreased.

The rule set that has the best performance twice in a row continues in the next generation. If the two cycles have their best measurements from different rule sets, then the size of the number of trials is increased and the original, non-mutated rule base is continued in the next generation. If a rule set shows repeated high performance, there is no reason to increase the size of the sample and spend the extra time and cycles. Then, the rule set is mutated using evolution and evaluated against the weather data using the rule evaluation technique. Two cycles of the subroutine are performed using the same number of mutations in an iterative fashion until all the test case samples are used or the best evaluation is found to be within an acceptable difference from the actual weather results.

The mutation process of the algorithm varies the number of features that are mutated depending on measurements of the algorithms progress towards a solution in order to optimize convergence of a global solution during progressive runs. The number of mutations starts with a small number (usually one) and if the progress toward a solution does not improve by a predetermined limit, such as the defined number of iterations or a timed limit, then the number of mutations implemented on the rule base is incremented by one or more. When the performance of the current mutated rule base improves with fewer iterations in comparison with the previous mutated rule base then the number of mutations can be decreased. If the algorithm uses more iterations to get an improvement than for any previous rule base the number of mutations increases. The following diagram is a general flowchart of how the software processes the algorithm. In this sense, the algorithm uses a hill-climbing technique to find the right number of mutations not un-similar to the one armed bandit algorithm [3].

This process is implemented by using data structures to store the number of iterations and their corresponding mutations, where previous mutations (set to a certain number) found improved performance. If the performance peak is found using a number of iterations that is similar to the number iterations it took to find improved performance from a previous run it will then use the stored number of mutations from the previous run for the next mutated set in the iterative process. The median values between iterations are computed.

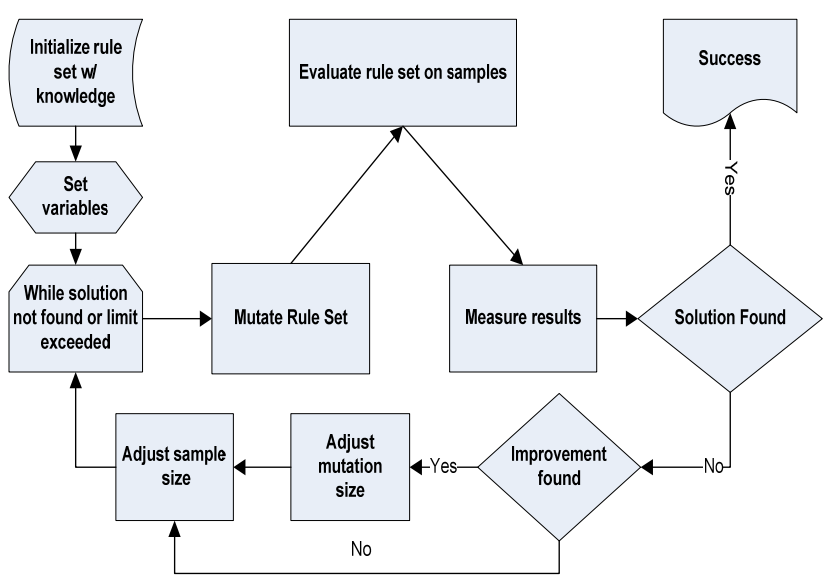

Figure 1: General Flow of Processing for Evolutionary Algorithm

If the number of iterations measured for the latest run's improvement is between one measurement of iterations and the median of the next largest measurement of iterations, it is rounded to the smaller number of iterations and the number of mutations used for the previous run will be used for mutating the next rule set in a new iteration.

Likewise, if the number of iterations measured for the latest run's improvement is between median value and a larger measurement of iterations stored in the data structure, it is rounded up to the larger number of iteration. The number of mutations is found by "hashing" into the data structure to find the number of mutations used for each number of iterations, where improvement was found in a generation. Thus, the number of mutations varies with the performance of the algorithms

\begin{tabular}{|l|l|l|l|l|l|l|}
\hline \# iterations & $\mathbf{2 4}$ & $\mathbf{3 0}$ & $\mathbf{4 6}$ & $\mathbf{5 2}$ & $\mathbf{6 8}$ & $\mathbf{7 8}$ \\
\hline \# mutations & $\mathbf{3}$ & $\mathbf{3}$ & $\mathbf{4}$ & $\mathbf{4}$ & $\mathbf{4}$ & $\mathbf{5}$ \\
\hline \begin{tabular}{l} 
Current Iteration \\
is 33: \\
$\begin{array}{l}\text { Choose \# of } \\
\text { mutations: } \mathrm{n}=3 \\
\text { for next run }\end{array}$ \\
\cline { 2 - 3 }
\end{tabular} \\
\hline
\end{tabular}


Table 1: An example of a data structure that stores the number of iterations it took to achieve improvement for a corresponding number of mutations to indicate a decision process for determining how many features are to be selected for mutation during an iteration.

The algorithm interpolates the number of mutations as it evolves improved performance. Large numbers of mutations, which take up a lot of time, are not needed when the algorithm converges on a solution. By continuously adjusting the number of mutations as the solution improves, processing time can be saved over the entire duration of the algorithm. Better solutions evolve with greater rapidity because the use of an excessive amount of mutation is minimized.

\section{Summary}

Many of the issues that are often confronted when using genetic or evolutionary algorithms can be addressed by varying the techniques of evaluating the progressive generations. As discussed, carefully varying the number of mutations and samples taken during the progression of the algorithm has a high potential to reduce the time it takes for the algorithm to converge to the optimal solution. Randomization subroutines and hill climbing are techniques to find optima in evolutionary algorithms. Hill climbing finds optima in the value of a function by making sequential small changes to the solution in order to find improvements in the algorithm. One issue with the hill-climbing technique is that it tends to only find local optima in the progression of the algorithm.

This topic has significant merit and potential use in the development of computational algorithms to analyze trends in dynamic systems. Progress in the research of evolutionary algorithms is advanced by applying different methods for the application of hill-climbing techniques and by varying the number of samples and mutations used in the progression of the algorithm. The issues of processing time and efficiency that are found in most evolutionary algorithms can be studied in detail and addressed. The results of this investigation could lead to the development of an evolutionary algorithm that, when applied, might offer a basis for critical operations in such fields as meteorology, financial planning, and scientific areas, which depend on the identification and prediction of trends.
This work was produced by U.S. government employees as part of their official duties and is not subject to copyright. It was supported, in part, by DTRA contract No. BA08MSB008 and is approved for public release with an unlimited distribution.

\section{References}

[1] Fogel, D. 2006. Evolutionary Computation, To Towards a New Philosophy of Machine Intelligence. John Wiley \& Sons, Inc., IEEE Press, 2006.

[2] Negnevitsky, M. Artificial Intelligence, $A$ Guide to Intelligent Systems, Peason Education, Unlimited, 2002. [3] Mitchell, M. An Introduction to Genetic Algorithms, A Bradford Book, MIT Press, Cambridge, 1997.

\section{Acknowledgements}

